\title{
The Liouville side of the Vortex
}

\author{
Giulio Bonelli ${ }^{\odot}$, Alessandro Tanzini and Jian Zhao \\ International School of Advanced Studies (SISSA) \\ via Bonomea 265, 34136 Trieste, Italy \\ and \\ INFN, Sezione di Trieste \\ and \\ ○.C.T.P. \\ Strada Costiera 11, 34014 Trieste, Italy
}

\begin{abstract}
We analyze conformal blocks with multiple (semi-)degenerate field insertions in Liouville/Toda conformal field theories an show that their vector space is fully reproduced by the four-dimensional limit of open topological string amplitudes on the strip with generic boundary conditions associated to a suitable quiver gauge theory. As a byproduct we identify the non-abelian vortex partition function with a specific fusion channel of degenerate conformal blocks.
\end{abstract}




\section{Contents}

1 Introduction $\quad 1$

$2 \mathrm{SU}(2)$ Vortices And Degenerate States $\quad 2$

2.1 General Setup . . . . . . . . . . . . . . . 2

$2.2 \mathrm{SU}(2)$ Vortices . . . . . . . . . . . . . . . . . . 5

$2.3 \mathrm{SU}(2)$ simple surface operators . . . . . . . . . . . . . . . 4

2.4 Relation To Open Topological String amplitudes . . . . . . . . . . . . . . 9

3 SU(N) Generalization $\quad 11$

$3.1 \mathrm{SU}(\mathrm{N})$ vortices ............................... 11

$3.2 \mathrm{SU}(\mathrm{N})$ Simple Surface Operators . . . . . . . . . . . . . . . . 13

3.3 Toda Fusion Rules From Quiver Gauge Theory . . . . . . . . . . . . . . . 14

4 Conclusions

\begin{tabular}{ll} 
A Appendix & 18 \\
\hline
\end{tabular}

A.1 Instanton Partition Functions . . . . . . . . . . . . . . . . . . . 18

A.2 Degeneration from bifundamental masses . . . . . . . . . . . . 19

A.3 Factorization formulae . . . . . . . . . . . . . . 222

\section{Introduction}

The connection between refined BPS counting in four dimensional $\mathcal{N}=2$ quiver gauge theories - namely Nekrasov partition functions [21] - and Logarithmic Conformal Field Theories on Riemann Surfaces [6], which was originally noticed in [3], opened a renewed perspective on both the areas. This correspondence can be studied via geometric engineering [18] where topological strings [24] can be used to exactly describe the BPS protected sectors of the gauge theory both at perturbative and non perturbative level and realizes the above program in M-theory [25, 16].

In this context, in [13, 12] the role of vortex counting was noticed and proposed to encode in a two dimensional field theoretic perspective the insertion of surface operators of the type discussed in [4, 19, 20]. The role of non-abelian vortices was exploded in [12] by relating their partition function [23] with instanton counting and topological string amplitudes.

The Liouville/Toda descriptions of some of these amplitudes with suitable boundary 
conditions were provided in terms of insertions of multiple degenerate fields. In presence of more than one insertion, the conformal blocks span a vector space whose dimension is fixed by the fusion rules of a generic primary with degenerate fields. One of the main results of this paper is to provide a full realization of the above in terms of topological string amplitudes with general boundary conditions. As a byproduct we identify the non-abelian vortex partition function with a specific fusion channel of the degenerate conformal block, different to the one considered so far in the literature.

Moreover, we realize the vortex counting problem as sub-counting instantons by showing how to relate the Nekrasov partition function and its vortex counterpart by a particular choice of mass parameters in an appropriately engineered gauge theory in four dimensions. On the gauge theory side, this boils down to consider surface operator insertions in a theory with a simpler quiver structure. On the AGT dual side, we notice that the above mass parameters assignments produces the insertion of degenerate fields in the Liouville/Toda CFT amplitudes. We study this correspondence in depth, reproduce some known results and embed them in a wider framework. In particular we show the correspondence between the fusion channel choice in the Liouville/Toda field theory side and the choice of possible surface operator insertions. The relation with topological strings, in the form of related strip amplitudes [2, 17], is also considered in full generality for the $S U(2)$ case and in some particular exemplificative ones for $S U(N)$.

We organize our paper as follows. In section 2 we calculate the CFT dual of $S U(2)$ vortex partition functions. In section 3 we extend the CFT dual for $S U(N)$ vortices and argue its validity for general strip amplitudes in section 4 . Section 5 contains our conclusion, while some technical details are left for the Appendix.

\section{$2 \mathrm{SU}(2)$ Vortices And Degenerate States}

\subsection{General Setup}

We start from two node $S U(2)$ theory with specific parameters. Its Liouville conformal block dual and brane construction is illustrated in Fig.1. Following the results of our previous paper [12], we will focus on the free field limit, $\epsilon_{+}:=\epsilon_{1}+\epsilon_{2}=0$. The parameters of this two node quiver are: $\mu_{1}, \mu_{2}$ are masses of antifundamental hypermultiples; $\mu_{3}, \mu_{4}$ are masses of fundamental hypermultiples; $m$ is the mass of bifundamental hypermultiplet and $\left(a_{1}, a_{2}\right)=(a,-a) ;\left(\tilde{a}_{1}, \tilde{a}_{2}\right)=(\tilde{a},-\tilde{a})$ are Coulomb branch parameters of the first and second gauge factor. On the conformal field theory side $\alpha_{1}, \alpha_{2}, \alpha_{3}$ are the external momenta in Liouville theory. When all parameters are generic, what we get is just the standard AGT correspondence between instanton partition functions of quiver gauge theories and conformal blocks with five operator insertions. When there are degenerate states, different fusion channels will give different results which also have different gauge theory interpretation as we will show in the following. For two node $S U(2)$ quiver theories there are two channels, one corresponding to $S U(2)$ vortex partition functions while the other to a simple surface operator as discussed in [19]. The general situation with the insertion of more degenerate 


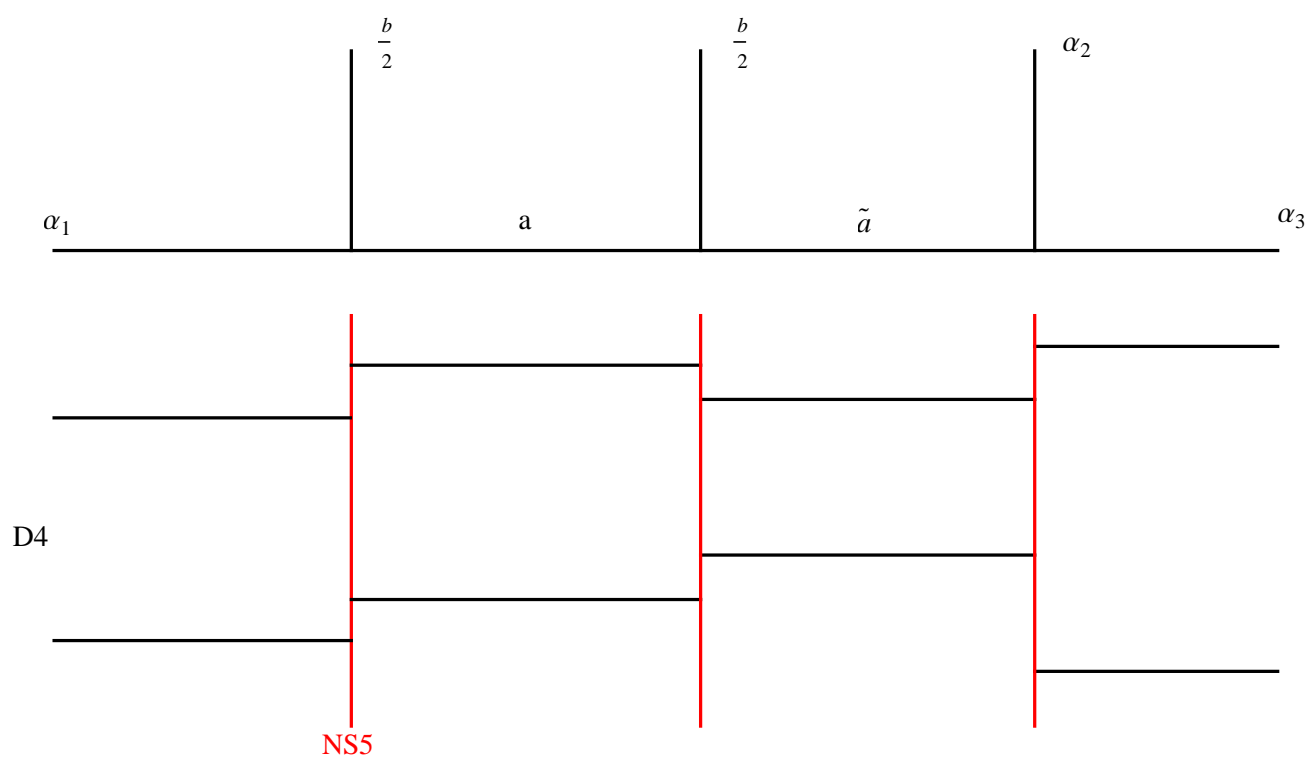

Figure 1: AGT relation between $S U(2)$ quiver gauge theory and CFT

fields is discussed in subsequent sections.

The standard AGT-relation [3] gives the following map between parameters:

$$
\begin{aligned}
\mu_{1} & =\alpha_{1}-\frac{\epsilon_{2}}{2} \\
\mu_{2} & =-\alpha_{1}-\frac{\epsilon_{2}}{2} \\
\mu_{3} & =\alpha_{2}+\alpha_{3} \\
\mu_{4} & =\alpha_{2}-\alpha_{3} \\
m & =-\frac{\epsilon_{2}}{2}
\end{aligned}
$$

The fusion rules of Liouville field theory imply that

$$
\begin{aligned}
\alpha_{1} & =a-s_{1} \frac{\epsilon_{2}}{2} \\
\tilde{a} & =a-s_{2} \frac{\epsilon_{2}}{2}
\end{aligned}
$$

where $s_{1}$ and $s_{2}$ are \pm 1 . This fixes the masses to

$$
\begin{gathered}
\mu_{1}=a-\left(s_{1}+1\right) \frac{\epsilon_{2}}{2} \\
\mu_{2}=-a+\left(s_{1}-1\right) \frac{\epsilon_{2}}{2}
\end{gathered}
$$

Let us remark that when the differences between Coulomb branch parameters and fundamental/bifundamental masses are linear in $\epsilon_{1}$ and $\epsilon_{2}$ the instanton partition function is 
largely simplified. To see this let us recall the contribution from antifundamental fields

$$
Z_{\text {antifund }}(m, a, Y)=\prod_{\alpha=1}^{2} \prod_{(i, j) \in Y_{\alpha}}\left(a_{\alpha}+m+\epsilon_{2}(j-i)\right)
$$

Where $a_{1}=a ; a_{2}=-a$, and $(i, j)$ are the box location in the Young tableaux. If we choose $s_{1}=-1$, then $\mu_{1}=a ; \mu_{2}=-a-\epsilon_{2}$. The above formula then implies that $Y_{2}=\emptyset$ and $Y_{1}$ to be a row. The other choice $s_{1}=1$ just exchanges the roles of $Y_{1}$ and $Y_{2}$. So the choice of fusion channel here is just a convention. What is really relevant is the choice of $s_{2}$.

Let us notice that bifundamental masses can transfer degeneration between adjacent gauge factors of a quiver theory. Indeed the contribution of bifundamental hypermultiples is:

$$
\begin{aligned}
& Z_{\text {bifund }}(m, a, \tilde{a}, Y, W)=\prod_{\alpha=1}^{2} \prod_{\beta=1}^{2} Z_{\text {bifund }}^{(\alpha, \beta)} \\
& Z_{\text {bifund }}^{(\alpha, \beta)}=\prod_{s \in Y_{\alpha}} \prod_{t \in W_{\beta}}\left(m_{\alpha, \beta}+\epsilon_{2}\left(A_{Y_{\alpha}}(s)+L_{W_{\beta}}(s)+1\right)\right)\left(m_{\alpha, \beta}-\epsilon_{2}\left(A_{W_{\beta}}(t)+L_{Y_{\alpha}}(t)+1\right)\right) \\
& m_{\alpha, \beta}:=a_{\alpha}-\tilde{a}_{\beta}-m
\end{aligned}
$$

From the second fusion relation in the diagram one gets

$$
\begin{aligned}
& m_{1,1}=\left(s_{2}+1\right) \frac{\epsilon_{2}}{2} \\
& m_{2,2}=\left(1-s_{2}\right) \frac{\epsilon_{2}}{2} \\
& m_{1,2}=2 a+\left(1-s_{2}\right) \frac{\epsilon_{2}}{2} \\
& m_{2,1}=-2 a+\left(1+s_{2}\right) \frac{\epsilon_{2}}{2}
\end{aligned}
$$

Moreover, AGT-correspondence implies that, up to a $U(1)$ factor which doesn't play any role here,

$$
\begin{aligned}
& Z_{\text {Quiver }}\left(a, \tilde{a}=a-s_{2} \frac{\epsilon_{2}}{2} ; \mu_{1}=a, \mu_{2}=-a-\epsilon_{2} ; \alpha_{2}+\alpha_{3}, \alpha_{2}-\alpha_{3}\right) \\
& =\mathcal{F}\left(a+\frac{\epsilon_{2}}{2}, \frac{-\epsilon_{2}}{2}, a, \frac{-\epsilon_{2}}{2}, a-s_{2} \frac{\epsilon_{2}}{2}, \alpha_{2}, \alpha_{3}\right)
\end{aligned}
$$

where the LHS is the instanton partition function of $S U(2)$ quiver gauge theory and the RHS is the conformal block of Liouville field theory.

In the following we will show that when $s_{2}=-1$ the quiver partition function in the above formula reduces to the $S U(2)$ vortex partition function, while when $s_{2}=1$, it corresponds to the $S U(2)$ simple surface operator. 


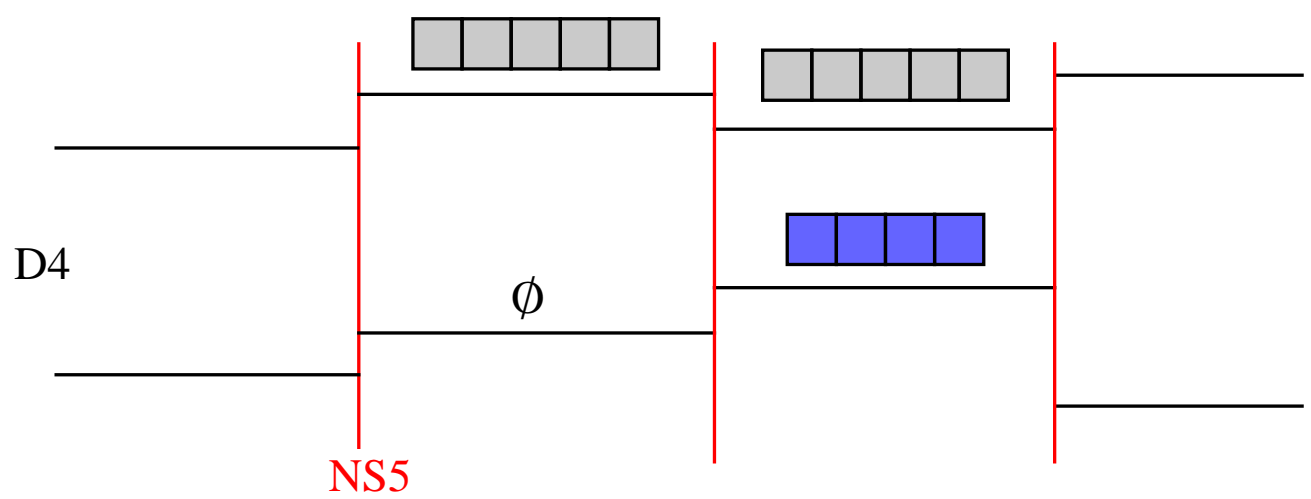

Figure 2: $S U(2)$ vortex from quiver theory

\section{$2.2 \quad \mathrm{SU}(2)$ Vortices}

Let us start investigating the case $s_{2}=-1$ where

$$
\begin{aligned}
& m_{1,1}=0 \\
& m_{2,2}=\epsilon_{2} \\
& m_{1,2}=2 a+\epsilon_{2} \\
& m_{2,1}=-2 a
\end{aligned}
$$

To start with, let's focus on $Z_{\text {bifund }}^{(1,1)}$ :

$$
Z_{\text {bifund }}^{(1,1)}=\prod_{s \in Y_{1}}\left(\epsilon_{2}\left(A_{Y_{1}}(s)+L_{W_{1}}(s)+1\right)\right) \prod_{t \in W_{1}}\left(-\epsilon_{2}\left(A_{W_{1}}(t)+L_{Y_{1}}(t)+1\right)\right)
$$

where:

$$
\prod_{t \in W_{1}}\left(-\epsilon_{2}\left(A_{W_{1}}(t)+L_{Y_{1}}(t)+1\right)\right)=\prod_{(i, j) \in W_{1}}-\epsilon_{2}\left(A_{W_{1}}(i, j)+L_{Y_{1}}(i, j)+1\right)
$$

From the discussion of the previous section we know that the choice of the fundamental mass parameter in (7) implies that $Y_{1}$ is a row diagram. Moreover, from the results in the Appendix, one gets that the bifundamental masses in (7) set also $W_{1}$ to be a row of the same length which we call $k_{1}$, see the Fig.2.

To simplify the formulæ, let's define some notations:

$$
(x)_{Y, W}:=\prod_{s \in Y} \prod_{t \in W}\left(x+\epsilon_{2}\left(A_{Y}(s)+L_{W}(s)+1\right)\right)\left(x-\epsilon_{2}\left(A_{W}(t)+L_{Y}(t)+1\right)\right)
$$

and

$$
\begin{aligned}
(x)_{Y} & :=(x)_{Y, \emptyset} \\
H_{Y} & :=(0)_{Y, \emptyset} \\
(x)_{k} & \left.:=(x)_{\emptyset,\left(1^{k}\right.}\right) \\
(x)_{k_{1}, k_{2}} & :=(x)_{\left(1^{k_{1}}\right),\left(1^{k_{2}}\right)}
\end{aligned}
$$


Let's calculate $Z_{\text {bifund }}^{(1,1)}$ explicitly

$$
Z_{\mathrm{bifund}}^{(1,1)}=\prod_{i=1}^{k_{1}} \epsilon_{2} i \prod_{j=1}^{k_{1}}-\epsilon_{2} j=\left(\epsilon_{2}\right)_{k_{1}}^{2}(-1)^{k_{1}}
$$

The contribution form $Z_{\text {bifund }}^{(2,2)}$ is instead

$$
Z_{\mathrm{bifund}}^{(2,2)}=\prod_{t \in W_{2}}-\epsilon_{2}\left(A_{W_{2}}(t)+L_{\emptyset}(t)\right)=\prod_{(i, j) \in W_{2}}-\epsilon_{2}(j-i-1)
$$

which is non zero only if $W_{2}$ is a row. Let's denote its length by $k_{2}$. Then

$$
Z_{\text {bifund }}^{(2,2)}=\left(\epsilon_{2}\right)_{k_{2}}
$$

By including the contributions from $Z_{\text {bifund }}^{(1,2)}$ and $Z_{\text {bifund }}^{(2,1)}$ we get the final formula

$$
Z_{\text {bifund }}=\left(\epsilon_{2}\right)_{k_{1}}^{2}(-1)^{k_{1}}\left(\epsilon_{2}\right)_{k_{2}}(-2 a)_{k_{1}}\left(2 a+\epsilon_{2}\right)_{k_{1}, k_{2}}
$$

The contribution from the anti-fundamental matter can be computed with analogous methods giving

$$
Z_{\text {antifund }}=\left(\epsilon_{2}\right)_{k_{1}}(-2 a)_{k_{1}}
$$

The generic contribution from the vector multiplets is

$$
\begin{aligned}
& Z_{\text {vect }}(a, Y)=\prod_{\alpha, \beta=1}^{2} Z_{\text {vect }}^{(\alpha, \beta)}(a, Y) \\
& Z_{\text {vect }}^{(\alpha, \beta)}(a, Y)=\prod_{s \in Y_{\alpha}} \prod_{t \in Y_{\beta}}\left(a_{a, \beta}+\epsilon_{2}\left(A_{Y_{\alpha}}(s)+L_{Y_{\beta}}(s)+1\right)\right)^{-1}\left(a_{\alpha, \beta}-\epsilon_{2}\left(A_{Y_{\beta}}(t)+L_{Y_{\alpha}}(t)+1\right)\right)^{-1} \\
& a_{\alpha, \beta}:=a_{\alpha}-a_{\beta}
\end{aligned}
$$

which reduces for the first node of our specific Young tableaux to

$$
Z_{\text {vect }}(a, Y)=\left(\left(\epsilon_{2}\right)_{k_{1}}(-2 a)_{k_{1}}\right)^{-2}
$$

The fundamental matter $Z_{\text {fund }}$ is in the standard form

$$
Z_{\text {fund }}=\left(\tilde{a}+\mu_{3}\right)_{k_{1}}\left(\tilde{a}+\mu_{4}\right)_{k_{2}}\left(-\tilde{a}+\mu_{3}\right)_{k_{1}}\left(-\tilde{a}+\mu_{4}\right)_{k_{2}}
$$

while the contribution from the second gauge factor of the quiver is:

$$
Z_{\text {vect }}(\tilde{a}, W)=\frac{(-1)^{k_{1}+k_{2}}}{\left(\epsilon_{2}\right)_{k_{1}}^{2}\left(\epsilon_{2}\right)_{k_{2}}^{2}\left(2 a+\epsilon_{2}\right)_{k_{1}, k_{2}}(-2 \tilde{a})_{k_{2}, k_{1}}}
$$

In summary, the total partition function of the quiver theory with specific choice of masses reads

$$
Z_{\text {Quiver }}\left(k_{1}, k_{2}\right)=\frac{(-1)^{k_{1}}\left(\tilde{a}+\mu_{3}\right)_{k_{1}}\left(\tilde{a}+\mu_{4}\right)_{k_{2}}\left(-\tilde{a}+\mu_{3}\right)_{k_{1}}\left(-\tilde{a}+\mu_{4}\right)_{k_{2}}}{\left(\epsilon_{2}\right)_{k_{1}}\left(\epsilon_{2}\right)_{k_{2}}(2 \tilde{a})_{k_{1}, k_{2}}}
$$


This, up-to a sign factor which can be absorbed in the vortex counting parameter coincides to ${ }^{1}$ the $S U(2)$ vortex partition function studied in [12]:

$$
Z_{\text {vortex }}^{\mathrm{SU}(2)}(\boldsymbol{k})=\frac{(-1)^{k_{2}}\left(a-m_{1}\right)_{k_{1}}\left(-a-m_{1}\right)_{k_{2}}\left(a-m_{2}\right)_{k_{1}}\left(-a-m_{2}\right)_{k_{2}}}{\left(\epsilon_{2}\right)_{k_{1}}\left(\epsilon_{2}\right)_{k_{2}}\left(a_{1,2}\right)_{k_{1}, k_{1}}}
$$

Notice that we should identify $m_{i}=-\mu_{i+2}$, and $\tilde{a}$ as $a$, since it is the second gauge factor that couples to hypermultiplets with generic masses.

To conclude the matching, notice that in the two nodes quiver theory, we have two parameters $q_{1}, q_{2}$ which are the exponential of the gauge couplings of the quiver theory. These are related to the vortex counting parameters $z_{1}, z_{2}$ of vortex partition functions as

$$
q_{1}^{k_{1}}\left(q_{2}\right)^{k_{1}+k_{2}}=\left(q_{1} q_{2}\right)^{k_{1}} q_{2}^{k_{2}}=z_{1}^{k_{1}} z_{2}^{k_{2}}
$$

From the CFT viewpoint $z_{i}$ are the insertion points of the degenerate fields.

\section{$2.3 \quad \mathrm{SU}(2)$ simple surface operators}

A natural question is to find what's the result in the other channel. As expected we find it is the result of [19]. So, let's now choose $s_{2}=1$, then

$$
\begin{aligned}
\tilde{a} & =a-\frac{\epsilon_{2}}{2} \\
m_{1,1} & =\epsilon_{2} \\
m_{2,2} & =0 \\
m_{1,2} & =2 a \\
m_{2,1} & =-2 a+\epsilon_{2}
\end{aligned}
$$

In this case, the contribution of the bifundamentals reads

$$
Z_{\text {bifund }}^{(1,1)}=\prod_{s \in Y_{1}}\left(\epsilon_{2}\left(A_{Y_{1}}(s)+L_{W_{1}}(s)+2\right)\right) \prod_{t \in W_{1}}\left(-\epsilon_{2}\left(A_{W_{1}}(t)+L_{Y_{1}}(t)\right)\right)
$$

where:

$$
\prod_{t \in W_{1}}\left(-\epsilon_{2}\left(A_{W_{1}}(t)+L_{Y_{1}}(t)\right)\right)=\prod_{(i, j) \in W_{1}}-\epsilon_{2}\left(A_{W_{1}}(i, j)+L_{Y_{1}}(i, j)\right)
$$

Using once again the results in the appendix, the bifundamental contribution

$$
Z_{\text {bifund }}^{(2,2)}=\prod_{t \in W_{2}}\left(-\epsilon_{2}\left(A_{W_{2}}(t)+L_{\emptyset}(t)+1\right)\right)=\prod_{t \in W_{2}}\left(-\epsilon_{2}(j-i)\right)
$$

is non vanishing only if $W_{2}=\emptyset$, see Fig.3.

\footnotetext{
${ }^{1}$ With respect to [12] we set $\hbar=-\epsilon_{2}$. These sign factors will be disregarded in the following without further notice.
} 


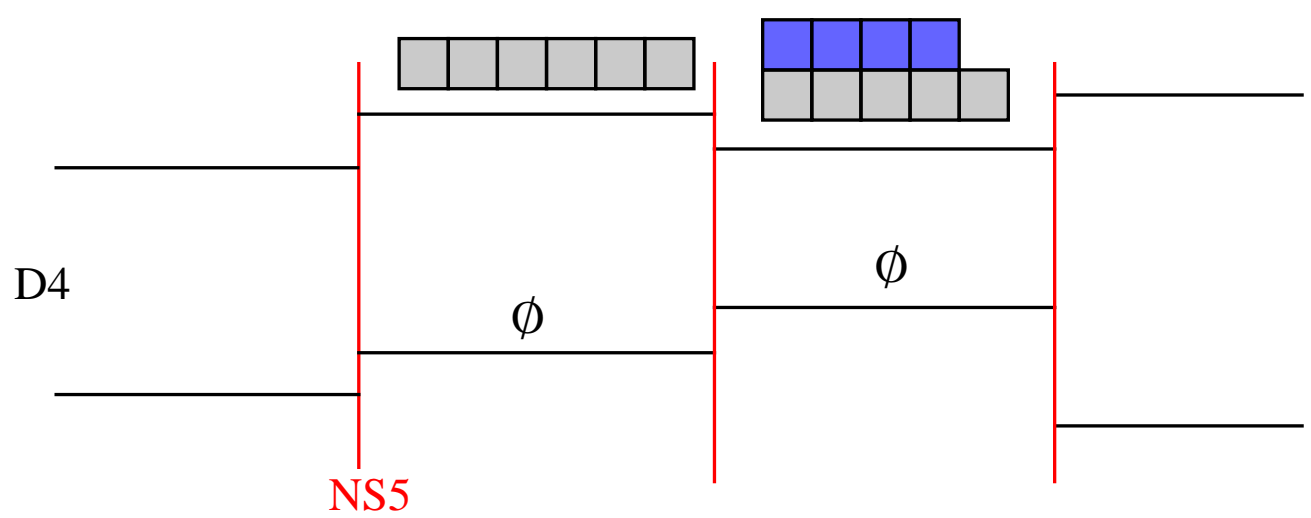

Figure 3: $S U(2)$ simple surface operators from quiver theory

Therefore, the bifundamental contributions are given by

$$
\begin{aligned}
& Z_{\text {bifund }}^{(1,1)}=H_{Y_{1}} H_{W_{1}}(-1)^{k_{1}} \\
& Z_{\text {bifund }}^{(2,2)}=1 \\
& Z_{\text {bifund }}^{(1,2)}=(-1)^{k_{1}+1}(-2 a)_{k_{1}+1} \\
& Z_{\text {bifund }}^{(2,1)}=(-1)^{k_{1}+k_{2}}\left(\tilde{a}_{1,2}\right)_{W_{1}}
\end{aligned}
$$

The contribution from the other factors can be analogously derived to be

$$
\begin{gathered}
Z_{\text {antifund }} Z_{\text {vect }}(a, Y)=\frac{1}{\left(\epsilon_{2}\right)_{k_{1}+1}(-2 a)_{k_{1}+1}} \\
Z_{\text {vect }}(\tilde{a}, W)=\frac{1}{\left(H_{W_{1}}\left(\tilde{a}_{1,2}\right)_{W_{1}}\right)^{2}} \\
Z_{\text {fund }}=\left(\tilde{a}-\mu_{3}\right)_{W_{1}}\left(\tilde{a}-\mu_{4}\right)_{W_{1}}
\end{gathered}
$$

and finally we get

$$
Z_{\text {Quiver }}\left(W_{1}\right)=\frac{(-1)^{k_{1}+k_{2}+1}\left(\tilde{a}-\mu_{3}\right)_{W_{1}}\left(\tilde{a}-\mu_{4}\right)_{W_{1}}}{H_{W_{1}}\left(\tilde{a}_{1,2}\right)_{W_{1}}}
$$

which is the partition function of $S U(2)$ simple surface operator [19]

$$
Z_{\text {simple surface }}=\frac{\left(a+m_{1}\right)_{W_{1}}\left(a+m_{2}\right)_{W_{1}}}{H_{W_{1}}\left(a_{1,2}\right)_{W_{1}}}
$$

Now the identification of parameters goes as

$$
q_{1}^{k_{1}+1}\left(q_{2}\right)^{k_{1}+k_{2}}=\frac{z_{1}}{z_{2}} z_{1}^{k_{1}} z_{2}^{k_{2}}
$$

As already noticed, $z_{i}$ are the insertion points of the degenerate fields. 

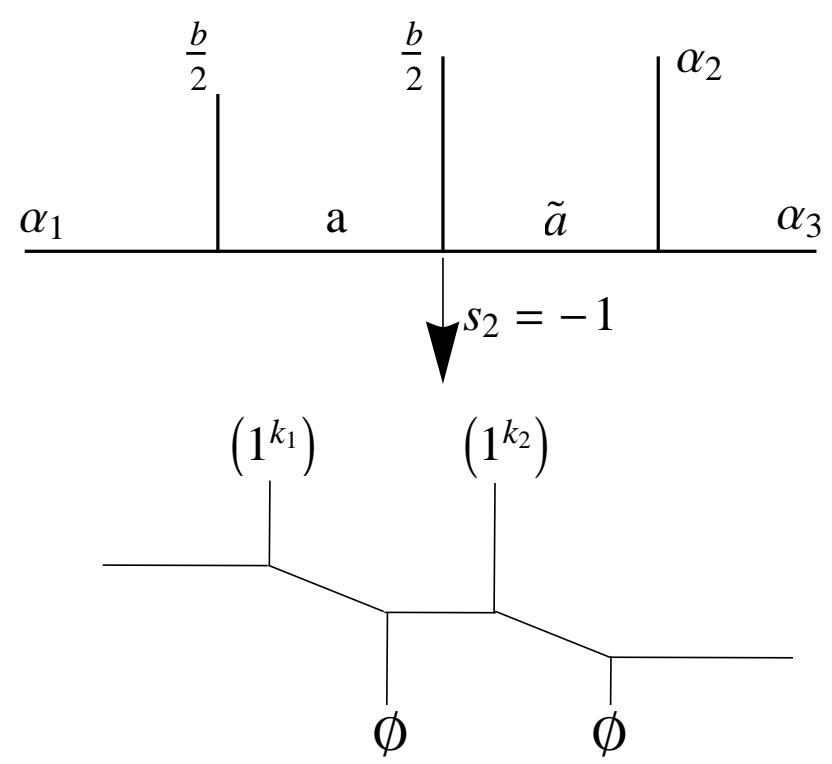

Figure 4: relation between $S U(2)$ vortex and CFT

\subsection{Relation To Open Topological String amplitudes}

The amplitudes discussed in the previous sections can be derived as four dimensional limits of Open Topological String amplitudes on the strip with suitable boundary conditions [19, 12]. The discussion of the previous section then provides the CFT interpretation of this class of strip amplitudes, as summarized in Fig.4 and Fig.5. Actually, this is the simplest situation. For example we can have more than two degenerate states, then does this story still holds? The answer is yes. From our previous calculations, we can deduce three general laws: (1)the number of nodes of the quiver equals the number of degenerate states. (2)the total number of rows of Young-tableaux increase by one when counting from left to right along the quiver of gauge theory nodes. (3) different fusion channels just tell us on which gauge factor of the quiver to associate an extra row in the partition. So if we have $n$ degenerate states, the corresponding quiver has $n$ nodes, and on each node there are two choices to add a new row. For convenience let's define a fusion vector $\mathfrak{V} \in \mathbb{Z}_{2}^{n}$, whose $i$-th component is 1 if we add a new row onto the partition attached to the first $D 4$ brane and 2 if to the second. For example, the non-abelian vortex partition function is associated to $\mathfrak{V}=(1,2)$, while the simple surface operator partition function is associated to $\mathfrak{V}=(1,1)$.

When we have $n$ degenerate states, the Young-tableaux on the final node are a couple $(Y, W)$ satisfying the constraint $n_{1}+n_{2}=n$, where $n_{1}, n_{2}$ are respectively the number of rows of $Y$ and of $W$. Hence we conclude that the four dimensional limit of the strip amplitudes of the form $A_{\{\emptyset, \emptyset\}}^{\{Y, W\}}$, that is with boundary conditions labeled by $Y$ and $W$, reproduces the full conformal block vector space including all the possible fusion channels. For example we can choose $\mathfrak{V}=(1, \ldots, 1,2, \ldots, 2)$, where there are $n_{1} 1$ 's and $n_{2} 2$ 's and can prove explicitly that for this choice of fusion vector our claim is correct, see Fig.6. 

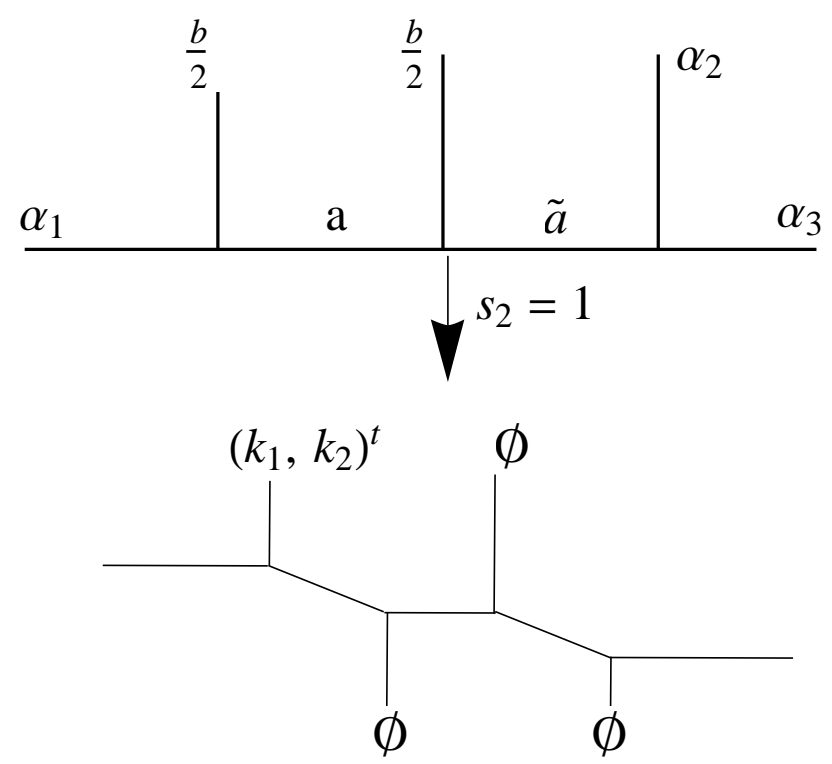

Figure 5: relation between $S U(2)$ simple surface operator and CFT
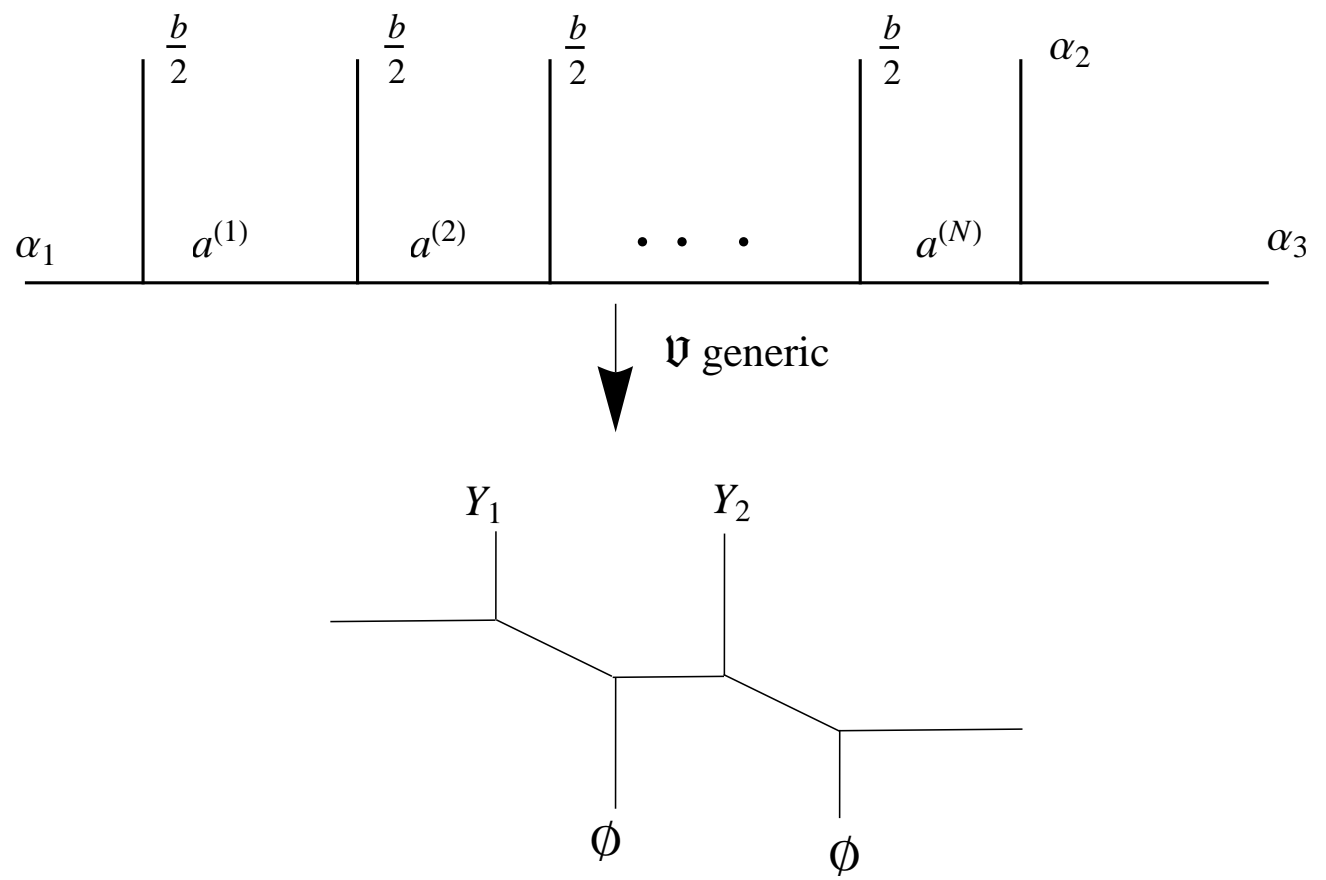

Figure 6: relation between $S U(2)$ strip amplitudes and CFT 

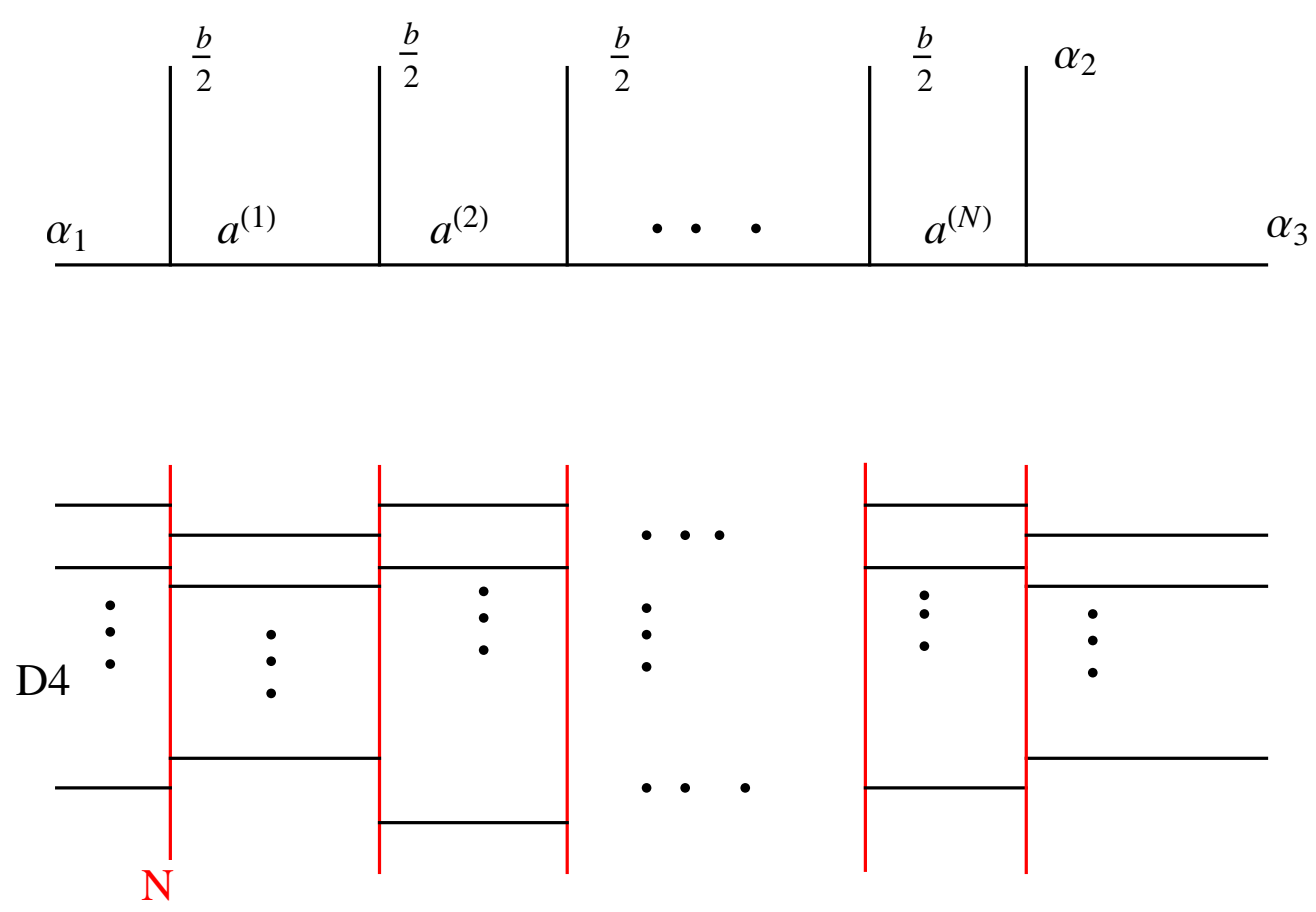

Figure 7: AGT relation between $S U(N)$ quiver gauge theory and CFT

\section{$3 \quad \mathrm{SU}(\mathrm{N})$ Generalization}

In the following we will give the natural generalization to $S U(N)$ theories. We know that the $S U(N)$ vortex partition function should have $N$ independent counting parameters, thus from the previous section's discussion we know that the associated $S U(N)$ quiver theory will have $N$ nodes. The quiver configuration reads as the brane construction illustrated in Fig.7.

\section{$3.1 \quad \mathrm{SU}(\mathrm{N})$ vortices}

The Young-tableaux configuration of quiver gauge theory corresponding to vortex partition function is such that at the $L$-th node the arrows of Young-tableaux are $Y^{(L)}=$ $\left(1^{k_{1}}, \ldots, 1^{k_{L}}, \emptyset, \ldots, \emptyset\right)$. This configuration can be obtained from a given bifundamental mass assignments as displayed in the following. We will see that this choice of masses correctly reproduces the fusion rules for Toda field theory.

Let us consider the $L$-th node of the quiver and calculate $Z_{L} Z_{L, L+1}$, where $Z_{L}$ is the vector contribution of the $L$-th node while $Z_{L, L+1}$ the corresponding bifundamental. Following the arguments in the Appendix, we can read out the $L$-th bifundamental mass to be

$$
m_{\alpha, \alpha}^{(L)}:=a_{\alpha}^{(L)}-a_{\alpha}^{(L+1)}-m_{L}=\delta_{\alpha, L+1} \epsilon_{2}
$$


Then the matrix of masses is given by

$$
m_{\alpha, \beta}^{(L)}=\left\{\begin{array}{cc}
a_{\alpha, \beta}^{(L)}=a_{\alpha, \beta}^{(L+1)} & \alpha \in[1, L] ; \beta=[1, L] \\
a_{\alpha, \beta}^{(L+1)} & \alpha \in[1, L] ; \beta \in[L+1, N] \\
a_{\alpha, \beta}^{(L)} & \alpha \in[L+1, N] ; \beta=[1, L]
\end{array}\right.
$$

We find it better to write $Z_{L}$ in three parts according to above mass matrix formula:

$$
Z_{L}^{-1}=\prod_{\alpha, \beta=1}^{L}\left(a_{\alpha, \beta}^{(L)}\right) k_{\alpha}, k_{\beta} \prod_{\alpha=1}^{L} \prod_{\beta=L+1}^{N}(-1)^{k_{\alpha}}\left(a_{\beta, \alpha}^{(L)}\right) k_{\alpha} \prod_{\beta=1}^{L} \prod_{\alpha=L+1}^{N}\left(a_{\alpha, \beta}^{(L)}\right) k_{\beta}
$$

Correspondingly, $Z_{L, L+1}$ read

$$
\begin{aligned}
Z_{L, L+1}= & \left\{\prod_{\alpha=1}^{L} \prod_{\beta=1}^{L}\left(m_{\alpha, \beta}^{(L)}\right)_{k_{\alpha}, k_{\beta}}\right\} \times \\
& \left\{\prod_{\alpha=1}^{L} \prod_{\beta=L+2}^{N}(-1)^{k_{\alpha}}\left(-m_{\alpha, \beta}^{(L)}\right)_{k_{\alpha}}\right\}\left\{\prod_{\alpha=1}^{L}\left(m_{\alpha, L+1}^{(L)}\right) k_{\alpha}, k_{L+1}\right\} \times \\
& \left\{\prod_{\alpha=L+1}^{N} \prod_{\beta=1}^{L}\left(m_{\alpha, \beta}^{(L)}\right) k_{\beta}\right\}\left\{\prod_{\alpha=L+1}^{N}\left(m_{\alpha, L+1}^{(L)}\right)_{k_{L+1}}\right\}
\end{aligned}
$$

Then we get:

$$
Z_{L} Z_{L, L+1}=\frac{\left\{\prod_{\alpha=1}^{L}\left(a_{\alpha, L+1}^{(L+1)}\right)_{k_{\alpha}, k_{L+1}}\right\}}{\left\{\prod_{\alpha=1}^{L}(-1)^{k_{\alpha}}\left(a_{L+1, \alpha}^{(L)}\right) k_{\alpha}\right\}}\left\{\prod_{\alpha=L+2}^{N}\left(a_{\alpha, L+1}^{(L+1)}\right)_{k_{L+1}}\right\}\left(\epsilon_{2}\right)_{k_{L+1}}
$$

The mass spectrum of the antifundamental hypermultiplets is assigned as

$$
\left(\mu_{1}, \mu_{2}, \ldots, \mu_{N}\right)=\left(-a_{1}^{(1)}-\epsilon_{2},-a_{2}^{(1)}, \ldots,-a_{N}^{(1)}\right)
$$

and the correspondent contribution to the instanton partition function is

$$
Z_{\text {antifund }}=\prod_{f=1}^{N} \prod_{i=1}^{k_{1}}\left(a_{1}^{(1)}+\mu_{f}+\epsilon_{2}(1-i)\right)=(-1)^{N k_{1}}\left(\epsilon_{2}\right)_{k_{1}} \prod_{i=2}^{N}\left(a_{i, 1}^{(1)}\right)_{k_{1}}
$$

Finally, the vector contribution of the last $N$-th node is

$$
Z_{N}^{-1}=\prod_{\alpha=1}^{N}\left(\epsilon_{2}\right)_{k_{\alpha}}^{2}(-1)^{k_{\alpha}} \prod_{\alpha<\beta}^{N}(-1)^{k_{\alpha}+k_{\beta}}\left(a_{\alpha, \beta}^{(N)}\right)_{k_{\alpha}, k_{\beta}}^{2}
$$

Then the instanton partition function of this quiver is:

$$
Z_{\text {Quiver }}=\frac{(-1)^{N k_{1}+\sum_{\alpha} k_{\alpha}} \prod_{\alpha, f=1}^{N}(-1)^{k_{\alpha}}\left(-a_{\alpha}^{(N)}+\mu_{f+N}\right) k_{\alpha}}{\prod_{\alpha=1}^{N}\left(\epsilon_{2}\right)_{k_{\alpha}} \prod_{\alpha<\beta}^{N}(-1)^{k_{\beta}}\left(a_{\alpha, \beta}^{(N)}\right) k_{\alpha}, k_{\beta}}
$$


Following the result of [12], and identifing $\hbar=-\epsilon_{2}$, the $S U(N)$ vortex partition function can be written as:

$$
\begin{aligned}
Z_{\mathrm{vortex}}^{\mathrm{SU}(N)} & =\sum_{\boldsymbol{k}} Z_{\mathrm{vortex}}^{\mathrm{SU}(N)}(\boldsymbol{k}) \prod_{i=1}^{N} z_{i}^{k_{i}} \\
Z_{\mathrm{vortex}}^{\mathrm{SU}(N)}(\boldsymbol{k}) & =\frac{\prod_{\alpha, f=1}^{N}(-1)^{k_{\alpha}}\left(-a_{\alpha}-m_{f}\right)_{k_{\alpha}}}{\prod_{\alpha=1}^{N}\left(\epsilon_{2}\right)_{k_{\alpha}} \prod_{\alpha<\beta}^{N}(-1)^{k_{\beta}}\left(a_{\alpha, \beta}\right)_{k_{\alpha}, k_{\beta}}}
\end{aligned}
$$

This can be identified with the quiver instanton partition function by setting $a_{\alpha}^{(N)}=a_{\alpha}$ and $m_{f}=-\mu_{N+f}$. The counting parameters $z_{i}$ are identified as

$$
\begin{aligned}
& \prod_{i=1}^{N} q_{i}^{\sum_{j=1}^{i} k_{j}}=\prod_{i=1}^{N} z_{i}^{k_{i}} \\
& z_{i}:=\prod_{j=i}^{N+1-i} q_{i}
\end{aligned}
$$

In conclusion, the instanton partition function of quiver gauge theory with $Y^{(L)}=$ $\left(1^{k_{1}}, . ., 1^{k_{L}}, \emptyset, \ldots, \emptyset\right)$ with parameters in formula $(32)$ and $(34)$ gives the $S U(N)$ vortex partition function.

\subsection{SU(N) Simple Surface Operators}

From the previous arguments we can argue that the four dimensional limit of the strip

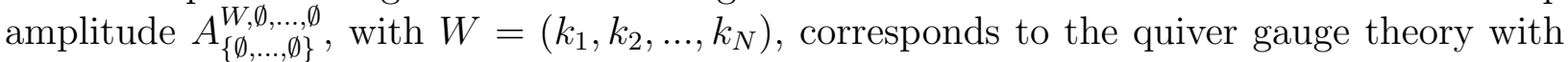
the following Young-tableaux assignment

$$
\begin{aligned}
Y^{(L)} & =\left(Y_{L}, \emptyset, \ldots, \emptyset\right) \\
Y_{L}^{t} & =\left(k_{1}+(N-L), k_{2}+(N-L), \ldots, k_{L}+(N-L)\right)
\end{aligned}
$$

The corresponding bifundamental masses can be obtained by following the arguments displayed in the appendix to be

$$
m_{\alpha, \alpha}^{(L)}=a_{\alpha}^{(L)}-a_{\alpha}^{(L+1)}-m_{L}=\delta_{\alpha, 1} \epsilon_{2}
$$

for the $L$-th node. The corresponding vector contribution for the $L$-th node is

$$
Z_{L}^{-1}=(-1)^{\left|Y_{L}\right|} H_{Y_{L}}^{2} \prod_{\beta=2}^{N}\left(a_{1, \beta}^{(L)}\right)_{Y_{L}} \prod_{\alpha=2}^{N}(-1)^{\left|Y_{L}\right|}\left(a_{1, \alpha}^{(L)}\right)_{Y_{L}}
$$

while the bifundamental is

$$
Z_{L, L+1}=\left(\epsilon_{2}\right)_{Y_{L}, Y_{L+1}} \prod_{\beta=2}^{N}\left(a_{1, \beta}^{(L)}\right)_{Y_{L}} \prod_{\alpha=2}^{N}(-1)^{\left|Y_{L+1}\right|}\left(a_{1, \alpha}^{(L+1)}\right)_{Y_{L+1}}
$$


so that

$$
\prod_{L=1}^{N-1} Z_{L} Z_{L, L+1}=\left\{\prod_{L=1}^{N-1} \frac{\left(\epsilon_{2}\right)_{Y_{L}, Y_{L+1}}}{(-1)^{\left|Y_{L}\right|} H_{Y_{L}}^{2}}\right\} \frac{\prod_{\alpha=2}^{N}(-1)^{\left|Y_{N}\right|}\left(a_{1, \alpha}^{(N)}\right)_{Y_{N}}}{\prod_{\alpha=2}^{N}(-1)^{\left|Y_{1}\right|}\left(a_{1, \alpha}^{(1)}\right)_{Y_{1}}}
$$

Using the result of the last Appendix, we can rewrite

$$
\left(\epsilon_{2}\right)_{Y_{L}, Y_{L+1}}=(-1)^{\left|Y_{L}\right|+L} H_{Y_{L}} H_{Y_{L+1}}
$$

and finally get

$$
\prod_{L=1}^{N-1} Z_{L} Z_{L, L+1}=(-1)^{\sum_{L=1}^{N-1} L} \frac{H_{Y_{N}} \prod_{\alpha=2}^{N}(-1)^{\left|Y_{N}\right|}\left(a_{1, \alpha}^{(N)}\right)_{Y_{N}}}{H_{Y_{1}} \prod_{\alpha=2}^{N}(-1)^{\left|Y_{1}\right|}\left(a_{1, \alpha}^{(1)}\right)_{Y_{1}}}
$$

Notice that, as in $S U(2)$ case, the spectrum of antifundamental hypermultiplets is fixed to be the same both for simple surface operator and nonabelian vortices. What distinguishes the different cases are the different fusion rules channels. The corresponding factors are then

$$
\begin{aligned}
Z_{\text {fund }} & =\prod_{f=1}^{N}\left(a_{1}^{(N)}-\mu_{f+N}\right)_{Y_{N}} \\
Z_{\text {antifund }} & =(-1)^{\left|Y_{1}\right|} H_{Y_{1}} \prod_{\alpha=2}^{N}\left(a_{1, \alpha}^{(1)}\right)_{Y_{1}} \\
Z_{N}^{-1} & =(-1)^{N\left|Y_{N}\right|} H_{Y_{N}}^{2} \prod_{\alpha=2}^{N}\left(a_{1, \alpha}\right)_{Y_{N}}^{2}
\end{aligned}
$$

which finally give

$$
Z_{\text {Quiver }}=(-1)^{\sum_{L=1}^{N} L+N\left|Y_{1}\right|+\left|Y_{N}\right|} \frac{\prod_{f=1}^{N}\left(a_{1}^{(N)}-\mu_{f+N}\right)_{Y_{N}}}{H_{Y_{N}} \prod_{\alpha=2}^{N}\left(a_{1, \alpha}^{(N)}\right)_{Y_{N}}} .
$$

This, after the identifications $\hbar=-\epsilon_{2}, a_{1}^{(N)}=a_{1}, m_{f}=-\mu_{f+N}$ and $\lambda=Y_{N}$, is the simple surface operator partition function discussed in [19] under the same counting parameters identification that we used in the last section.

\subsection{Toda Fusion Rules From Quiver Gauge Theory}

In this subsection we show how to derive fusion rules of semidegenerate states of Toda field theory from our construction. Let's concentrate on the L-th node of the quiver and recall the diagonal part of the mass assignment

$$
m_{\alpha, \alpha}^{(L)}:=a_{\alpha}^{(L)}-a_{\alpha}^{(L+1)}-m_{L}
$$


By denoting $\mathrm{m}_{L}=m_{L}(1,1, \ldots, 1)$, being a vector of $N$ entries all equal to $m_{L}$, we can write the above formula as

$$
\mathrm{a}^{(L)}-\mathrm{a}^{(L+1)}=\mathrm{m}^{(L)}-\mathrm{m}_{L}
$$

where $\mathrm{a}^{(L)}$ denotes the vector of internal momenta at the $L$-th node and $\mathrm{m}^{(L)}$ the vector of diagonal entries of the mass matrix at the $L$-node. Actually, for this assignment of external momenta, Toda fusion rules have $N$ channels. For the $\mathrm{i}$-th channel $\mathrm{m}^{(L)}=\epsilon_{2} u_{i}=$

$\epsilon_{2}\left(u_{1}-\sum_{j=1}^{i-1} e_{j}\right)$. Where $u_{i}$ is the unit vector in the $\mathrm{i}$-th direction in $\mathbb{R}^{N}$ and $e_{j}:=u_{j}-u_{j+1}$ are the simple roots of the $\mathfrak{s l}_{N}$ algebra. Then we have

$$
\mathrm{a}^{(L)}-\mathrm{a}^{(L+1)}=\epsilon_{2}\left(u_{1}-\sum_{j=1}^{i-1} e_{j}\right)-\mathrm{m}_{L}=\epsilon_{2}\left(u_{1}-\frac{\mathrm{m}_{L}}{\epsilon_{2}}\right)-\epsilon_{2} \sum_{j=1}^{i-1} e_{j}
$$

If we set $\mathrm{m}_{L}=\epsilon_{2} \frac{1}{N}(1,1, \ldots, 1)$, then

$$
\mathrm{a}^{(L)}-\mathrm{a}^{(L+1)}=\epsilon_{2}\left(-\omega_{1}\right)-\epsilon_{2} \sum_{j=1}^{i-1} e_{j}
$$

where $\omega_{1}$ is the highest weight of the fundamental representation of $\mathfrak{s l}_{N}$. The above formula can be recognized as the fusion rule calculated in [15].

For $S U(N)$ N nodes quiver, we can have $N$ semidegenerate states, for each one of them we have $N$ channels. We can use a $N$-dimensional vector of integer entries $\mathfrak{V}$ to denote the choice of the fusion channels. The fusion vector $\mathfrak{V}$ is built as follows: if on the $L$-th node we choose $k$-th channel, namely $m_{\alpha, \alpha}^{(L)}=\epsilon_{2} \delta_{\alpha, k}$, then the corresponding $L$-th component of $\mathfrak{V}$ is set equal to $k$. For example for the $S U(N)$ vortex $\mathfrak{V}_{\text {vortex }}=(1,2, \ldots, N)$, while for $S U(N)$ simple surface operator, $\mathfrak{V}_{\text {simplesurface }}=(1,1, \ldots, 1)$.

The relation with the four dimensional limit of strip amplitudes goes as in the $S U(2)$ as depicted in Figs.8,9 and Fig.10.

Notice that the four dimensional limit of strip amplitudes correspond to conformal blocks with only two independent external momenta, and one independent internal momentum. The number of degenerate states inserted in the conformal block corresponds to the total number of rows of the Young tableaux parametrizing the open string boundary conditions. This suggests that in order to have arbitrary boundary conditions one should consider conformal blocks with an arbitrary number of degenerate field insertions. Since we know that the full instanton partition function can be obtained by gluing two strip amplitudes with generic boundary conditions, this would provide a conformal field theory picture of this operation. From the CFT viewpoint, the infinite number of degenerate insertions could condense in a line operator [14] which could be used to glue the two CFT amplitudes to obtain the full result.

\section{Conclusions}

In this paper we studied the relation between non-abelian vortex partition functions and Liouville/Toda conformal field theories, by showing how to reproduce these partition functions 

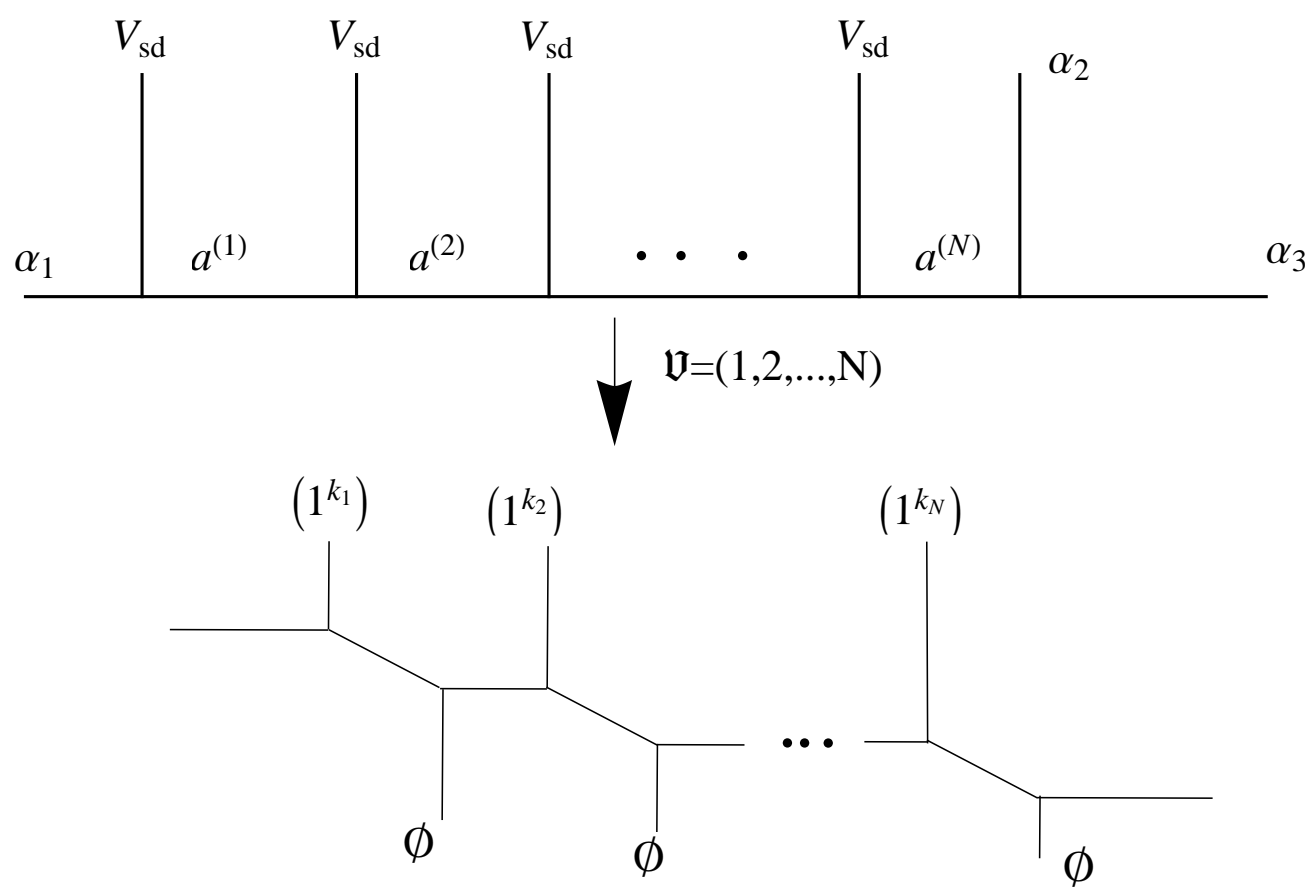

Figure 8: relation between $S U(N)$ vortex and CFT
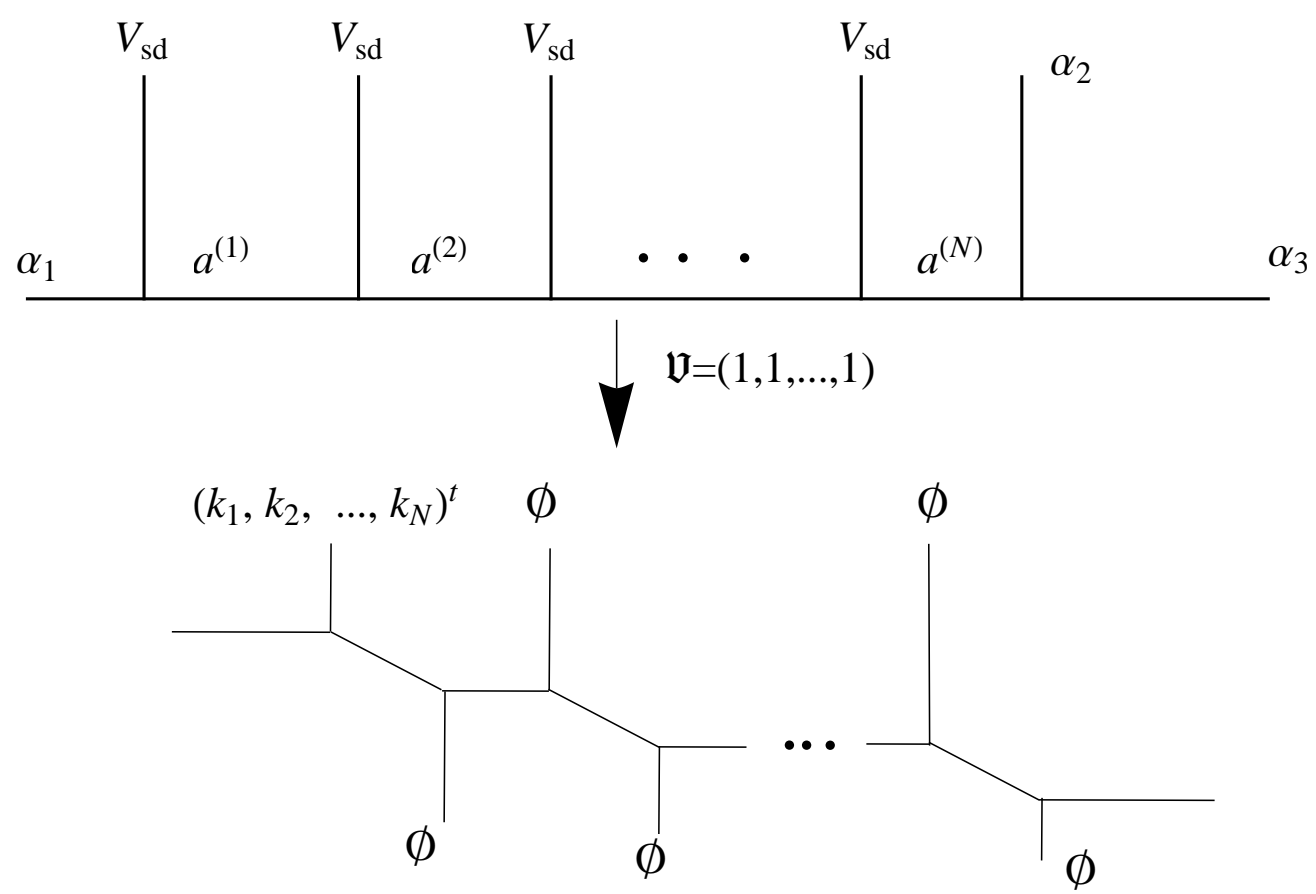

Figure 9: relation between $S U(N)$ simple surface operators and CFT 

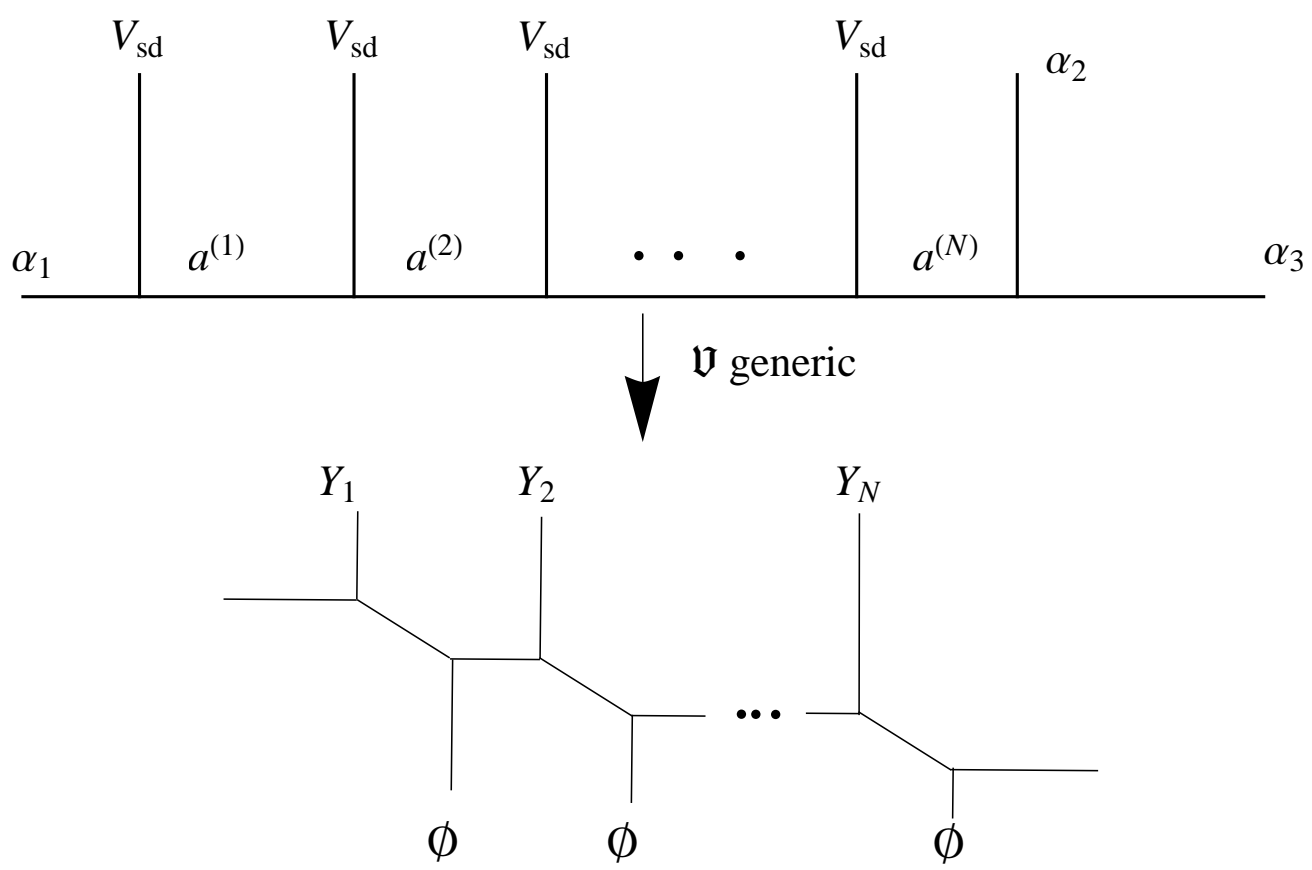

Figure 10: relation between $S U(N)$ strip amplitudes and CFT

from conformal blocks with degenerate field insertions. Moreover, we performed a general analysis using geometric engineering for open topological strings and found that there is a much richer structure in this correspondence which arises by identifying the full vector space of degenerate conformal blocks with the four dimensional limit of open topological strings amplitudes on a strip with general boundary conditions. A natural generalisation of this approach would be to analyse the full refined topological string amplitudes from the CFT viewpoint possibly along the lines of [5]. Another interesting venue is the investigation of the correspondence with integrable systems and their quantization [11, 10, 1] and in particular their relevance in vortex counting and more in general for open topological string amplitudes on the strip.

As discussed in [13, 12, vortices partition functions arise in the classical limit of four dimensional gauge theories with surface operator insertions. The analysis presented in this paper should then provide the classical limit of multiple surface operator insertions. In particular the approach of quiver gauge theories that we presented can be generalised to encompass also the four-dimensional instanton corrections. This should be completed with a description of the moduli space of instantons with multiple defect insertions.

An analogous analysis could be performed for surface operators in gauge theories on ALE spaces which have been recently related to para Liouville/Toda conformal CFTs [8, 22, 9, 7]. In this case the relevant vortex moduli space should be obtained as a lagrangian submanifold of the moduli space of instantons on ALE spaces.

Acknowledgements We thank H. Kanno, K. Maruyoshi and S. Pasquetti for interesting 
discussions and comments. G.B. and Z.J. are partially supported by the INFN project TV12. A.T. is partially supported by PRIN "Geometria delle varieta algebriche e loro spazi di moduli" and the INFN project PI14.

\section{A Appendix}

\section{A.1 Instanton Partition Functions}

Let us consider the instanton partition function of a linear quiver with $\mathrm{N}$ nodes. The corresponding brane construction has $N+2$ sets of D4-branes and $N+1 N S 5$ branes. We will focus on unrefined limit $\epsilon_{1}=-\epsilon_{2}$.

$$
Z_{\text {Quiver }}=Z_{\text {fund }} Z_{\text {antifund }} Z_{N} \prod_{i=1}^{N-1} Z_{i} Z_{i, i+1}
$$

$Z_{\text {fund }}$ and $Z_{\text {antifund }}$ are the contributions from fundamental and antifundamental hypermultiplets. $Z_{i}$ is the contribution from the $i$-th gauge factor, while $Z_{i, i+1}$ is the contribution from the $i$-th bifundamental hyper. These depend on the following parameters

$$
\begin{aligned}
a_{i}^{(N)} & =\text { the } \mathrm{i} \text {-th Coulomb branch parameter of the N-th gauge factor. } \\
m_{i} & =\text { the i-th mass of bifundamental hypermultiplet } \\
\mu_{i} & =\left\{\begin{array}{c}
\text { masses of antifundamental hypermultiplets } \\
\text { masses of fundamental hypermultiplets }
\end{array} i \in[N+1, N]\right. \\
Y^{(i)} & : \text { the arrow of Young-tableaux on the i-th node. }
\end{aligned}
$$

More explicitly:

$$
\begin{aligned}
Z_{\text {antifund }}\left(a^{(1)}, \mu, Y^{(1)}\right) & =\prod_{f=1}^{N} \prod_{\alpha=1}^{N} \prod_{(i, j) \in Y_{\alpha}^{(1)}}\left(a_{\alpha}^{(1)}+\mu_{f}+\epsilon_{2}(j-i)\right) \\
Z_{\text {fund }}\left(a^{(N)}, \mu, Y^{(N)}\right) & =\prod_{f=1}^{N} \prod_{\alpha=1}^{N} \prod_{(i, j) \in Y_{\alpha}^{(N)}}\left(a_{\alpha}^{(N)}-\mu_{f+N}+\epsilon_{2}(j-i)\right)
\end{aligned}
$$

The $L$-th bifundamental hypermultiplet contribution is:

$$
\begin{aligned}
Z_{L, L+1}= & \prod_{\alpha=1}^{N} \prod_{\beta=1}^{N} Z_{L, L+1}^{(\alpha, \beta)} \\
Z_{L, L+1}^{(\alpha, \beta)}= & \prod_{s \in Y_{\alpha}^{(L)}}\left(m_{\alpha, \beta}^{(L)}+\epsilon_{2}\left(A_{Y_{\alpha}^{(L)}}(s)+L_{Y_{\beta}^{(L+1)}}(s)+1\right)\right) \\
& \prod_{t \in Y_{\beta}^{(L+1)}}\left(m_{\alpha, \beta}^{(L)}-\epsilon_{2}\left(A_{Y_{\beta}^{(L+1)}}(t)+L_{Y_{\alpha}^{(L)}}(t)+1\right)\right) \\
m_{\alpha, \beta}^{(L)}:= & a_{\alpha}^{(L)}-a_{\beta}^{(L+1)}-m_{L}
\end{aligned}
$$



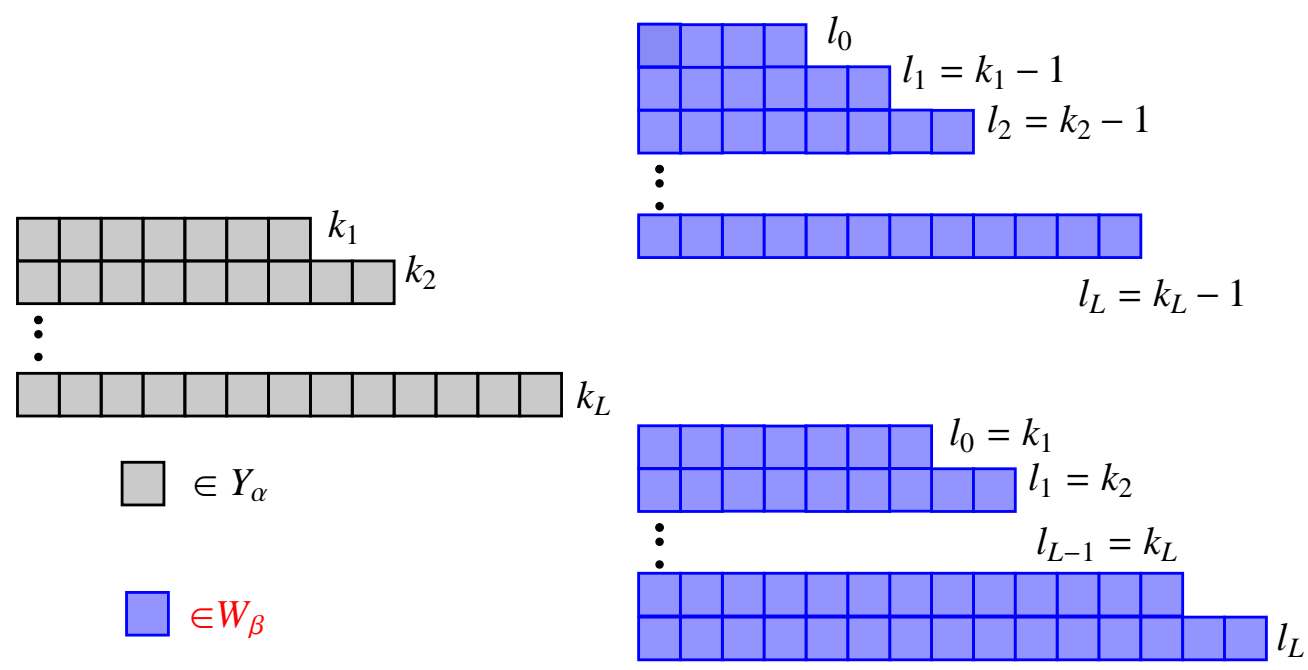

Figure 11: Relation between $Y_{\alpha}$ and $W_{\beta}$ when $m_{\alpha, \beta}=\epsilon_{2}$

Then consider contribution from s.

The $L$-th gauge factor contribution is:

$$
\begin{aligned}
Z_{L}= & \prod_{\alpha=1}^{N} \prod_{\beta=1}^{N} Z_{L}^{(\alpha, \beta)} \\
\left(Z_{L}^{(\alpha, \beta)}\right)^{-1}= & \prod_{s \in Y_{\alpha}^{(L)}}\left(a_{\alpha, \beta}^{(L)}+\epsilon_{2}\left(A_{Y_{\alpha}^{(L)}}(s)+L_{Y_{\beta}^{(L)}}(s)+1\right)\right) \\
& \prod_{t \in Y_{\beta}^{(L)}}\left(a_{\alpha, \beta}^{(L)}-\epsilon_{2}\left(A_{Y_{\beta}^{(L)}}(t)+L_{Y_{\alpha}^{(L)}}(t)+1\right)\right)
\end{aligned}
$$

For a Young-tableau $Y$, one box $s$ has coordinates $(i, j)$, where $i$ counts the number of columns and $j$ counts the number of rows. Then the arm and leg of $s$ relative to another Young-tableau $W$, are defined as $A_{W}(s):=W_{i}-j ; L_{W}(s):=W_{j}^{t}-i$. Where $W^{t}$ is the dual partition of $W .|Y|:=\sum_{i} Y_{i}$. We call a partition of the form $\left(1^{k}\right)$ a row partition of length $k$, and a partition of the form $(k)$ a column partition.

\section{A.2 Degeneration from bifundamental masses}

Let us state our results and then prove them. The claim is that when $m_{\alpha, \beta}=0, W_{\beta}=Y_{\alpha}$ and when $m_{\alpha, \beta}=\epsilon_{2}, W_{\beta}$ has one row more than that of $Y_{\alpha}$. In this situation, if we suppose $Y_{\alpha}$ has $L$ rows with lengths $k_{1} \leq k_{2} \leq \ldots \leq k_{L}$ and $W_{\beta}$ had $L+1$ rows with lengths $l_{0} \leq l_{1} \leq \ldots \leq l_{L}$, then for $1 \leq i \leq L$ either $k_{i}=l_{i-1}$ or $k_{i}=l_{i}+1$. Please refer to Fig.11 for a pictorial illustration. Let's start from the simpler case $m_{\alpha, \beta}=0$.

$$
Z_{\text {bifund }}^{(\alpha, \beta)}=\prod_{s \in Y_{\alpha}}\left(\epsilon_{2}\left(A_{Y_{\alpha}}(s)+L_{W_{\beta}}(s)+1\right)\right) \prod_{t \in W_{\beta}}\left(-\epsilon_{2}\left(A_{W_{\beta}}(t)+L_{Y_{\alpha}}(t)+1\right)\right)
$$


Let's suppose $Y_{\alpha}^{t}=\left(k_{1}, k_{2}, \ldots, k_{L}\right) ; W_{\beta}^{t}=\left(l_{1}, l_{2}, \ldots, l_{M}\right)$. We will proceed in our proof by induction from the top row to the bottom.

If $M>L$, then the result is non vanishing only if $L_{Y_{\alpha}}(t)+1=L_{\emptyset}(t)+1=-i+1 \neq 0$. The same argument applies for $L \leq M$, so that we stay with $M=L$.

The first induction step is when $t$ is on the top row of $W_{\beta}$, so that $A_{W_{\beta}}(t)=0$, and $L_{Y_{\alpha}}(t)+1=1+k_{1}-i_{t}$

$$
\left\{\begin{array}{c}
L_{Y_{\alpha}}(t)+1=1+k_{1}-i_{t} \neq 0 \\
i_{t} \in\left[1, l_{1}\right]
\end{array} \Longrightarrow k_{1} \geq l_{1}\right.
$$

Similarly for the contribution from $s \in Y_{\alpha}$, we get $l_{1} \geq k_{1}$, implying $k_{1}=l_{1}$. Suppose now $k_{i}=l_{i}$ when $i \leq p-1$ and let's prove that $k_{p}=l_{p}$.

1. $i_{t} \in\left[1, l_{1}\right], A_{W_{\beta}}(t)=p-1$

$$
\left\{\begin{array}{c}
L_{Y_{\alpha}}(t)+p=p+k_{p}-i_{t} \neq 0 \\
i_{t} \in\left[1, l_{1}\right]
\end{array} \Longrightarrow k_{p} \geq l_{1}-(p-1)\right.
$$

2. when $i_{t} \in\left[l_{1}+1, l_{2}\right] A_{W_{\beta}}(t)=p-2$

$$
\left\{\begin{array}{c}
L_{Y_{\alpha}}(t)+1+p-2=p-1+k_{p}-i_{t} \neq 0 \\
i_{t} \in\left[1+l_{1}, l_{2}\right]
\end{array} \Longrightarrow k_{p} \leq l_{1}+1-p \text { or } k_{p} \geq l_{2}-p+2\right.
$$

Since $k_{1}=l_{1}$, then $k_{p} \neq l_{1}+1-p$ and $k_{p} \geq l_{2}-p+2$. By iterating this procedure we find $k_{p} \geq l_{p}$, and symmetrically $l_{p} \geq k_{p}$, namely $l_{p}=k_{p}$. This ends the proof of the first statement.

Now let us concentrate on $m_{\alpha, \beta}=\epsilon_{2}$

$$
Z_{\text {bifund }}^{(\alpha, \beta)}=\prod_{s \in Y_{\alpha}}\left(\epsilon_{2}\left(A_{Y_{\alpha}}(s)+L_{W_{\beta}}(s)+2\right)\right) \prod_{t \in W_{\beta}}\left(-\epsilon_{2}\left(A_{W_{\beta}}(t)+L_{Y_{\alpha}}(t)\right)\right)
$$

It is easy to show that $W_{\beta}$ can have at most one row more than $Y_{\alpha}$. Suppose that $Y_{\alpha}^{t}=$ $\left(k_{1}, k_{2}, \ldots, k_{L}\right) ; W_{\beta}^{t}=\left(l_{0}, l_{1}, l_{2}, \ldots, l_{M}\right)$ and apply induction again from top to bottom.

When $t$ is on the top row of $W_{\beta}$ there is no constraint for the length $l_{0}$.

When $t$ is on the next to top row of $W_{\beta}$ then:

1. for $i_{t} \in\left[1, l_{0}\right]$, in this case $A_{W_{\beta}}(t)=1$

$$
\left\{\begin{array}{c}
L_{Y_{\alpha}}(t)+1=1+k_{1}-i_{t} \neq 0 \\
i_{t} \in\left[1, l_{0}\right]
\end{array} \Longrightarrow k_{1} \geq l_{0}\right.
$$

2. for $i_{t} \in\left[l_{0}+1, l_{1}\right]$, in this case $A_{W_{\beta}}(t)=0$

$$
\left\{\begin{array}{c}
L_{Y_{\alpha}}(t)=k_{1}-i_{t} \neq 0 \\
i_{t} \in\left[1+l_{0}, l_{1}\right]
\end{array} \Longrightarrow k_{1} \leq l_{0} \text { or } k_{1} \geq l_{1}+1\right.
$$


so we have $k_{1}=l_{0}$ or $k_{1} \geq l_{1}+1$.

Let us consider now the contribution from $Y_{\alpha}$. When $s$ is on the top row of $Y_{\alpha}, A_{Y_{\alpha}}(s)=0$ and we get

$$
\left\{\begin{array}{c}
L_{W_{\beta}}(s)+2=l_{1}-i_{s}+2 \neq 0 \\
i_{s} \in\left[1, k_{1}\right]
\end{array} \Longrightarrow l_{1} \geq k_{1}-1\right.
$$

so $k_{1}=l_{0}$ or $k_{1} \geq l_{1}+1 \cap l_{1} \geq k_{1}-1 \Longrightarrow k_{1}=l_{0}$ or $k_{1}=l_{1}+1$. Now suppose that for $i \leq p-1$, we have $l_{i}=k_{i}-1$ or $l_{i-1}=k_{i}$. Then

1. for $i_{t} \in\left[1, l_{0}\right], A_{W_{\beta}}(t)=p$ and

$$
\left\{\begin{array}{c}
L_{Y_{\alpha}}(t)+p=p+k_{p}-i_{t} \neq 0 \\
i_{t} \in\left[1, l_{0}\right]
\end{array} \Longrightarrow k_{p} \geq l_{0}-p+1\right.
$$

2. for $i_{t} \in\left[l_{0}+1, l_{1}\right], A_{W_{\beta}}(t)=p-1$

$$
\left\{\begin{array}{c}
L_{Y_{\alpha}}(t)+p-1=p-1+k_{p}-i_{t} \neq 0 \\
i_{t} \in\left[1+l_{0}, l_{1}\right]
\end{array} \Longrightarrow k_{p} \leq l_{0}+1-p \text { or } k_{p} \geq l_{1}+2-p\right.
$$

so we have $k_{p}=l_{0}+1-p$ or $k_{p} \geq l_{1}+2-p$ By iterating this procedure we get : $k_{p}=$ $l_{0}+1-p$ or $k_{p}=l_{1}+2-p, \ldots$, or $k_{p}=l_{p-1}$ or $k_{p} \geq l_{p}+1$. From the induction assumption we have $k_{p} \geq l_{p}+1$ or $k_{p}=l_{p-1}$.

Let us now consider the contribution from $s \in Y_{\alpha}$.

1. for $i_{s} \in\left[1, k_{1}\right], A_{Y_{\alpha}}(s)=p-1$

$$
\left\{\begin{array}{c}
L_{W_{\beta}}(s)+1+p=l_{p}-i_{s}+1+p \neq 0 \\
i_{s} \in\left[1, k_{1}\right]
\end{array} \Longrightarrow l_{p} \geq k_{1}-p\right.
$$

2. for $i_{s} \in\left[k_{1}+1, k_{2}\right], A_{Y_{\alpha}}(s)=p-2$

$$
\left\{\begin{array}{c}
L_{W_{\beta}}(s)+p=l_{p}-i_{s}+p \neq 0 \\
i_{s} \in\left[k_{1}+1, k_{2}\right]
\end{array} \Longrightarrow l_{p} \leq k_{1}-p \text { orl }_{p} \geq k_{2}-p+1\right.
$$

so we find $l_{p}=k_{1}-p$ or $l_{p} \geq k_{2}-p+1$. By iterating the procedure we find $l_{p}=k_{1}-p$ or $l_{p}=$ $k_{2}-p+1, \ldots$, or $l_{p}=k_{p-1}-2$ or $l_{p} \geq k_{p}-1$. From the induction assumption it follows that $l_{p} \geq k_{p}-1$.

Finally, combining the results from $W_{\beta}$ and $Y_{\alpha}$, we have $: k_{p}=l_{p}+1$ or $k_{p}=l_{p-1}$, which is what we wanted to prove. 


\section{A.3 Factorization formulae}

$$
\begin{aligned}
& \text { When } Y_{L}^{t}=\left(l_{1}+1, l_{2}+1, \ldots, 1+l_{L}\right) Y_{L+1}^{t}=\left(l_{0}, l_{1}, l_{2}, \ldots, l_{L}\right) \quad\left(l_{i} \leq l_{i+1}\right) \\
& \qquad \begin{array}{l}
\left(\epsilon_{2}\right)_{Y_{L}, Y_{L+1}}=\prod_{(i, j) \in Y_{L}}\left(\epsilon_{2}+\epsilon_{2}\left(A_{Y_{L}}(i, j)+L_{Y_{L+1}}(i, j)+1\right)\right) \\
=\prod_{(a, b) \in Y_{L+1}}\left(\epsilon_{2}-\epsilon_{2}\left(A_{Y_{L+1}}(a, b)+L_{Y_{L}}(a, b)+1\right)\right) \\
\\
=\prod_{(a, j) \in Y_{L}}\left(\epsilon_{2}+\epsilon_{2}\left(A_{Y_{L}}(i, j)+L_{Y_{L}}(i, j)\right)\right) \\
=\prod_{(a, b) \in Y_{L+1} \backslash Y_{L}}\left(\epsilon_{Y_{L}} \prod_{(a, b) \in Y_{L+1} \cap Y_{L}}-\epsilon_{Y_{L+1}}(a, b) \prod_{(a, b) \in Y_{L+1} \backslash Y_{L}} h_{Y_{L+1}}(a, b)\right. \\
=
\end{array}
\end{aligned}
$$

Similarly , when $Y_{L}^{t}=\left(l_{1}, l_{2}, \ldots, l_{L}\right)$ and $Y_{L+1}^{t}=\left(l_{1}, l_{2}, \ldots, l_{L+1}\right) \quad\left(l_{i} \leq l_{i+1}\right)$

$$
\begin{aligned}
\left(\epsilon_{2}\right)_{Y_{L}, Y_{L+1}} & =\prod_{(i, j) \in Y_{L}}\left(\epsilon_{2}\left(\left(A_{Y_{L}}(i, j)+1\right)+L_{Y_{L+1}}(i, j)+1\right)\right) \\
& =\prod_{(a, b) \in Y_{L+1}}\left(-\epsilon_{2}\left(\left(A_{Y_{L+1}}(a, b)-1\right)+L_{Y_{L}}(a, b)+1\right)\right) \\
& \prod_{(i, j) \in Y_{L}} h_{Y_{L+1}}(i, j) \prod_{(a, b) \in Y_{L}}(-1)^{\left|Y_{L}\right|} h_{Y_{L}}(a, b) \\
& =\prod_{(a, b) \in Y_{L+1} \backslash Y_{L}}\left(-\epsilon_{2}\left(\left(A_{Y_{L+1}}(a, b)-1\right)+L_{Y_{L}}(a, b)+1\right)\right)
\end{aligned}
$$

\section{References}

[1] M. Aganagic, M. C. N. Cheng, R. Dijkgraaf, D. Krefl and C. Vafa, arXiv:1105.0630 [hep-th].

[2] M. Aganagic, A. Klemm, M. Marino and C. Vafa, Commun. Math. Phys. 254, 425 (2005) arXiv:hep-th/0305132.

[3] L. F. Alday, D. Gaiotto and Y. Tachikawa, "Liouville Correlation Functions from Fourdimensional Gauge Theories," arXiv:0906.3219 [hep-th]. 
[4] L. F. Alday, D. Gaiotto, S. Gukov, Y. Tachikawa and H. Verlinde, "Loop and surface operators in $\mathrm{N}=2$ gauge theory and Liouville modular geometry," arXiv:0909.0945 [hepth].

[5] H. Awata, B. Feigin, A. Hoshino, M. Kanai, J. Shiraishi and S. Yanagida, "Notes on Ding-Iohara algebra and AGT conjecture," arXiv:1106.4088 [math-ph].

[6] A. A. Belavin, A. M. Polyakov and A. B. Zamolodchikov, Nucl. Phys. B 241 (1984) 333.

[7] A. Belavin, V. Belavin and M. Bershtein, arXiv:1106.4001 [hep-th].

[8] V. Belavin and B. Feigin, arXiv:1105.5800 [hep-th].

[9] G. Bonelli, K. Maruyoshi and A. Tanzini, arXiv:1106.2505 [hep-th].

[10] G. Bonelli, K. Maruyoshi and A. Tanzini, arXiv:1104.4016 [hep-th].

[11] G. Bonelli and A. Tanzini, Phys. Lett. B 691 (2010) 111 [arXiv:0909.4031 [hep-th]]. J. Teschner, arXiv:1005.2846 [hep-th].

[12] G. Bonelli, A. Tanzini and J. Zhao, "Vertices, Vortices and Interacting Surface Operators," arXiv: 1102.0184v1 [hep-th].

[13] T. Dimofte, S. Gukov and L. Hollands, arXiv:1006.0977 [hep-th].

[14] N. Drukker, D. Gaiotto and J. Gomis, JHEP 1106 (2011) 025 arXiv:1003.1112 [hepth]].

[15] V. A. Fateev and A. V. Litvinov, "Correlation functions in conformal Toda field theory I," JHEP 0711 (2007) 002 arXiv:0709.3806 [hep-th]].

[16] D. Gaiotto, "N=2 dualities," arXiv:0904.2715 [hep-th].

[17] A. Iqbal and A. K. Kashani-Poor, Adv. Theor. Math. Phys. 10 (2006) 317 arXiv:hepth/0410174.

[18] S. H. Katz, A. Klemm and C. Vafa, Nucl. Phys. B 497 (1997) 173 arXiv:hepth/9609239.

[19] C. Kozcaz, S. Pasquetti and N. Wyllard, JHEP 1008 (2010) 042 arXiv:1004.2025 [hepth]].

[20] A. Marshakov, A. Mironov and A. Morozov, Teor. Mat. Fiz. 164 (2010) 1:3 arXiv:1011.4491 [hep-th]]; H. Kanno and Y. Tachikawa, JHEP 1106, 119 (2011); M. Taki, arXiv:1007.2524 [hep-th]. D. Gaiotto, arXiv:0911.1316 [hep-th]. H. Awata, H. Fuji, H. Kanno, M. Manabe and Y. Yamada, "Localization with a Surface Operator, Irregular Conformal Blocks and Open Topological String," arXiv:1008.0574 [hep-th]. K. Maruyoshi and M. Taki, Nucl. Phys. B 841, 388 (2010) arXiv:1006.4505 [hep-th]]. 
U. Bruzzo, W. y. Chuang, D. E. Diaconescu, M. Jardim, G. Pan and Y. Zhang, "Dbranes, surface operators, and ADHM quiver representations," arXiv:1012.1826 [hep-th]. A. Mironov and A. Morozov, J. Phys. A 43 (2010) 195401 [arXiv:0911.2396 [hep-th]]. A. Mironov and A. Morozov, JHEP 1004 (2010) 040 arXiv:0910.5670 [hep-th]].

[21] N. A. Nekrasov, Adv. Theor. Math. Phys. 7, 831 (2004) arXiv:hep-th/0206161

[22] T. Nishioka and Y. Tachikawa, arXiv:1106.1172 [hep-th].

[23] S. Shadchin, JHEP 0708, 052 (2007) arXiv:hep-th/0611278.

[24] E. Witten, Commun. Math. Phys. 118, 411 (1988).

[25] E. Witten, "Solutions of four-dimensional field theories via M-theory," Nucl. Phys. B 500 (1997) 3 [arXiv:hep-th/9703166]. 